\title{
Modeling of Electromagnetic Fields in Railway Engineering Structures
}

\author{
Buyakova N.V. \\ Department of Power Supply of Industrial Enterprises \\ Angarsk State Technical University \\ Angarsk, the Russian Federation \\ e-mail: bn_900@mail.ru
}

\author{
Zakaryukin V.P. \\ Department of Transport Electricity \\ Irkutsk State Transport University \\ Irkutsk, the Russian Federation \\ e-mail: zakar49@mail.ru
}

\author{
Kryukov A.V. \\ Department of Transport Electricity \\ Irkutsk State Transport University \\ Department of Electrical Supply \\ Irkutsk National Research and Development University \\ Irkutsk, the Russian Federation \\ e-mail: and_kryukov@mail.ru
}

\begin{abstract}
The article presents the method and results of modeling of electromagnetic safety conditions in traction networks on railway sections passing through engineering structures: galleries, bridges, tunnels. Metal structures of engineering structures significantly change spatial distribution of electromagnetic field strengths. This is explained by the presence of grounded conductive objects, transforming tunnels, galleries and bottom-road bridges into confined spaces, as well as with the remoteness of the ground surface on top-road bridges.

The methods for modeling the modes of railway power supply systems, developed by Irkutsk State Transport University, allow the use up to several hundred wires in the models. Thus, it is possible to consider engineering structures (bridges, embankments, galleries and tunnels) with a set of grounded wires arranged in such way that the gaps between them are significantly less than the distance to the observation points.

The results of electromagnetic field modeling in railway engineering structures show that the top-road bridge has field strengths higher than those in the open space at the height of 1.8 $m$ from the railway track. Tunnel, a bottom-road bridge and a gallery are characterized by less strength and lower fluxes of electromagnetic energy. This is due to the proximity to the contact network of shielding metal elements of these engineering structures. The transfer of energy by the electromagnetic field in tunnels, galleries and bottom-road bridges occurs in a confined space between the contact network and nearby grounded structures.
\end{abstract} safety

Keywords-railway; engineering structures; electromagnetic

\section{INTRODUCTION}

Electromagnetic fields (EMF) created by high-voltage or high-current power transmission lines can generate various types of electrical interferences, causing malfunction of electrical and electronic devices [1,2], lead to ignition of flammable substances, and fatal accidents. Such cases may take place during working on disconnected power lines and communications when the induced voltages affect personnel.

The complex of electromagnetic processes in a given region of space is characterized by the term "electromagnetic environment". The main characteristics of these processes are the electric and magnetic field strengths.

Under conditions of the electrified railway, it is difficult to obtain experimental data corresponding to EMF maximum strength levels, therefore it is recommended to perform an analysis of the electromagnetic environment in railway power supply systems on the basis of mathematical modeling [3-9]. As a rule, EMF strengths are calculated by numerical methods of field theory, based on the first group of Maxwell's formulas, conformal transformations, and finite differences [2]. The use of such methods is characterized by the great complexity of initial data preparation, as well as the significant labor efforts associated with the study of specialized computer programs.

\section{MODELING TECHNIQUE}

These methods of modeling the railway power supply systems (RPSS) are based on the use of lattice-type equivalent circuits (LEC), which have fully-meshed topology. For LEC, the following formalized definition can be written:

$$
\text { TEC }: \text { hub } \bigcup c o n, \forall i, j \subset h u b \rightarrow \text { con }_{i, j} \subset \text { con }
$$

where TEC is LEC designation; $h u b$ is a set of LEC nodes; con is a set of LEC branches .

The basic elements that form a three-phase/single-phase network can be divided into two groups: 
- elements for power transmission: overhead and cable power lines, bus bars, electrical traction networks (ETN);

- conversion elements, which include various design transformers.

These devices can be considered as static multi-conductor elements, which are represented by the set of wires or windings with electromagnetic couplings [10]. This approach allows implementing electromagnetic safety analysis technique, which has the following features [3]:

- consistency, i.e. possibility for modeling electromagnetic fields, taking into account the properties and characteristics of a complex RPPS and the power supply system;

- universality, which allows modeling of power transmission lines and electrical traction networks of various designs;

- complexity, ensured by the combination of mode calculations and EMF determination;

- adequacy to the external environment, achieved by accurate account of underlying surface profile, underground communications, artificial transport structures.

The analysis of electromagnetic safety conditions is carried out in two stages.

1. The first stage includes the determination of RPSS mode or their complex corresponding to the movement of the train set in the selected time interval.

2. After determination of RPSS mode, it is possible to calculate electromagnetic fields created by the multi-wire transmission line or electrical traction network that make up modeled system.

If $Y$-axis direction is vertical and $X$ axis is perpendicular to the axis of the railroad, so that the $Z$ axis is directed opposite to flow of contact network, the electric field strengths of $N$ wires system at the point with $(x, y)$ coordinates are calculated by the following formulas:

$$
\begin{gathered}
\dot{E}_{Y}=-\frac{1}{\pi \varepsilon_{0}} \sum_{i=1}^{N} \dot{\tau}_{i} \frac{y_{i}\left[\left(x-x_{i}\right)^{2}-y^{2}+y_{i}^{2}\right]}{\xi} ; \\
\dot{E}_{X}=\frac{2}{\pi \varepsilon_{0}} \sum_{i=1}^{N} \dot{\tau}_{i} \frac{\left(x-x_{i}\right) y y_{i}}{\xi},
\end{gathered}
$$

where $\xi=\left[\left(x-x_{i}\right)^{2}+\left(y+y_{i}\right)^{2}\right]\left[\left(x-x_{i}\right)^{2}+\left(y-y_{i}\right)^{2}\right] ; \quad \dot{\tau}_{i}$ is $i$ wire charge per length unit that is determined from the first group of Maxwell's formulas:

$$
\dot{\mathbf{T}}=\mathbf{A}^{-1} \cdot \dot{\mathbf{U}},
$$

where $\dot{\mathbf{U}}=\left[\begin{array}{lll}\dot{U}_{1} & \ldots & \dot{U}_{N}\end{array}\right]^{\mathrm{T}}$ is column-vector of wire voltages against the earth; $\dot{\mathbf{T}}=\left[\begin{array}{lll}\dot{\tau}_{1} & \ldots & \dot{\tau}_{N}\end{array}\right]^{\mathrm{T}}$ is column-vector of wire charges, $\mathbf{A}$ is symmetric matrix of potential coefficient,

$$
\begin{gathered}
\alpha_{i i}=\frac{1}{2 \pi \varepsilon_{0}} \ln \frac{2 y_{i}}{r_{i}}, \\
\alpha_{i j}=\frac{1}{2 \pi \varepsilon_{0}} \ln \frac{\sqrt{\left(x_{i}-x_{j}\right)^{2}+\left(y_{i}+y_{j}\right)^{2}}}{\sqrt{\left(x_{i}-x_{j}\right)^{2}+\left(y_{i}-y_{j}\right)^{2}}},
\end{gathered}
$$

where $x_{i}$ and $y_{i}$ are coordinates of the location of the wire $i$ with radius $r_{i}$ above the ground ( $\mathrm{y}=0$ corresponds to the surface of the flat earth), $\varepsilon_{0}$ is electric constant.

Vertical and horizontal components of the magnetic field strength, created by all wires, are calculated by the following formulas:

$$
\begin{aligned}
\dot{H}_{X} & =\frac{1}{2 \pi} \sum_{i=1}^{N} \dot{I}_{i} \frac{y-y_{i}}{\left(x_{i}-x\right)^{2}+\left(y_{i}-y\right)^{2}} ; \\
\dot{H}_{Y} & =-\frac{1}{2 \pi} \sum_{i=1}^{N} \dot{I}_{i} \frac{x-x_{i}}{\left(x_{i}-x\right)^{2}+\left(y_{i}-y\right)^{2}},
\end{aligned}
$$

where $\dot{I}_{i}$ is currents flowing on the wires of electrical traction network and conductors simulating grounded objects.

Considering the results of determining the components $\dot{E}_{X}, \dot{E}_{Y}, \dot{H}_{X}, \dot{H}_{Y}$, the amplitude values of the strengths $E_{\mathrm{MAX}}, H_{\mathrm{MAX}}$ are calculated. Moreover, the density of active power flux density can be determined from expression:

$$
\begin{aligned}
& \Pi_{0}=E_{X} H_{Y} \cos \left(\psi_{E x}-\psi_{H y}\right)- \\
& -E_{Y} H_{X} \cos \left(\psi_{E y}-\psi_{H x}\right)
\end{aligned},
$$

where $\psi_{E x}, \psi_{E y}, \psi_{H x}, \psi_{H y}$ are the phase angles of the components $\dot{E}_{X}, \dot{E}_{Y}, \dot{H}_{X}, \dot{H}_{Y}$.

The described methods for modeling modes of railway power supply systems and realized Fazonord software complex allow the use of up to several hundred wires in models [10]. This makes it possible to simulate the conductive constructions of artificial structures by sets of grounded wires arranged in such a way that the gaps between them are much less than the distance to the observation point. The next section presents the results of modeling the electromagnetic environment at these objects.

\section{RESULTS OF MODELING}

Metal elements of railway engineering structures (tunnels, bridges, galleries) significantly change the pattern of spatial distribution of EMF strength. This is explained by the presence of grounded conductive objects, transforming tunnels, galleries and bottom-road bridges into confined spaces, as well as with the remoteness of the ground surface on top-road bridges. To analyze the difference of EMF in engineering structures from EMF in the open space, studies 
were carried out for the objects, cross sections of which are shown in Fig. 1-3.
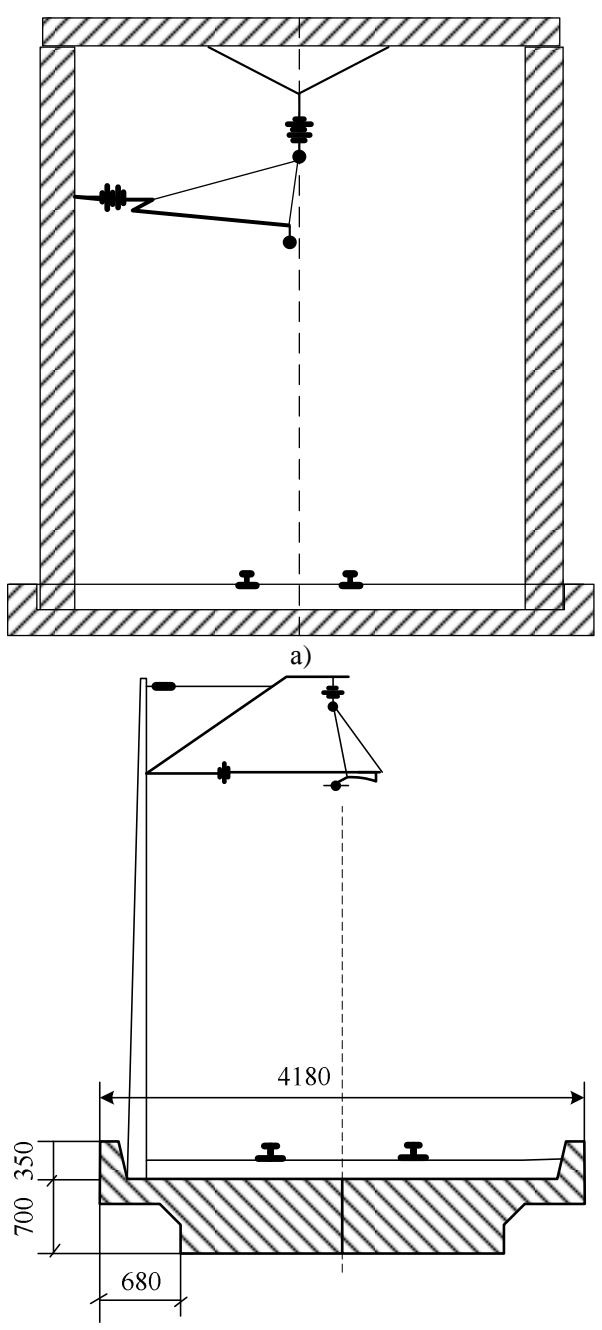

b)

Fig. 1. Typical cross-sections of railway bridges: a - bottom-road bridge; $b$ - top-road bridge

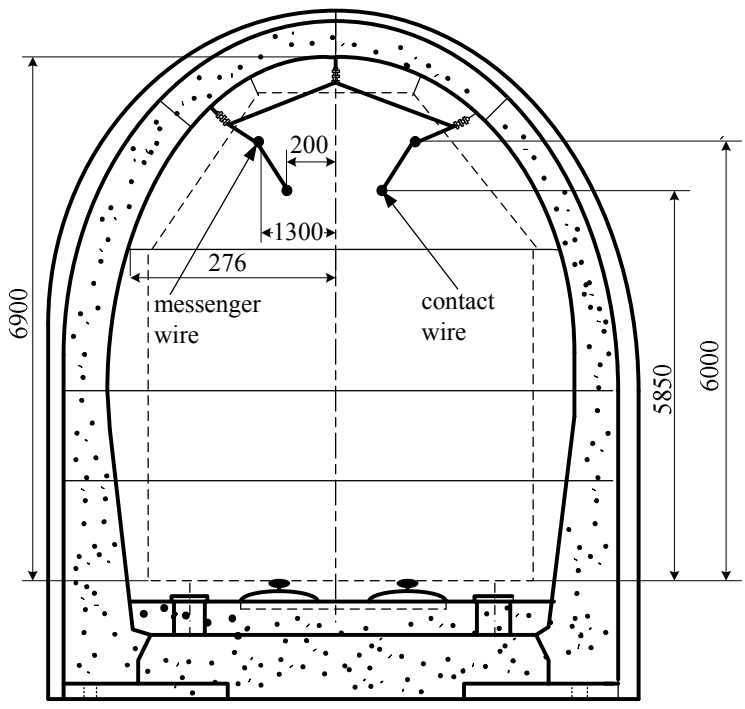

Fig. 2. Typical cross section of tunnel

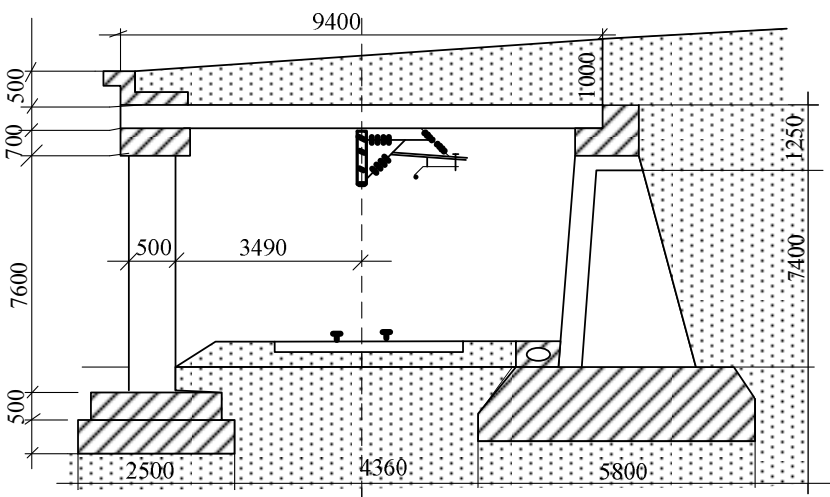

Fig. 3. Typical cross section of the gallery

Models of these structures are realized with the help of sets of thin grounded wires in the Fazonord software package. Coordinates of conducting parts and conductors simulating metal structures are shown in Fig. 4. In the calculations, potential of the rail threads and grounded conductors was assumed to be equal to zero (but considering induced currents).

View of Fazonord models is shown in Fig. 5.
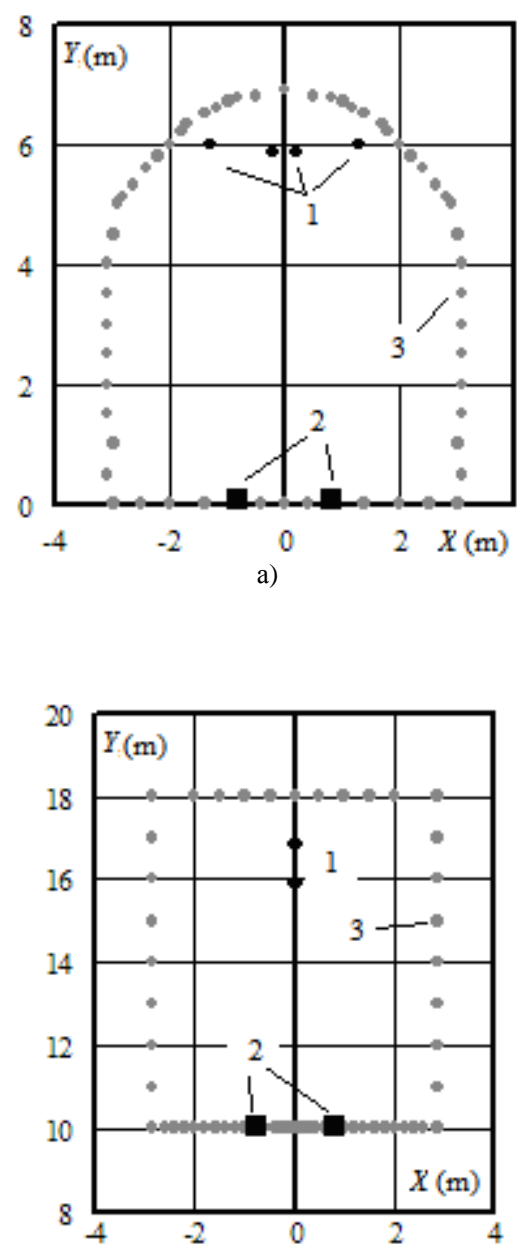

b) 


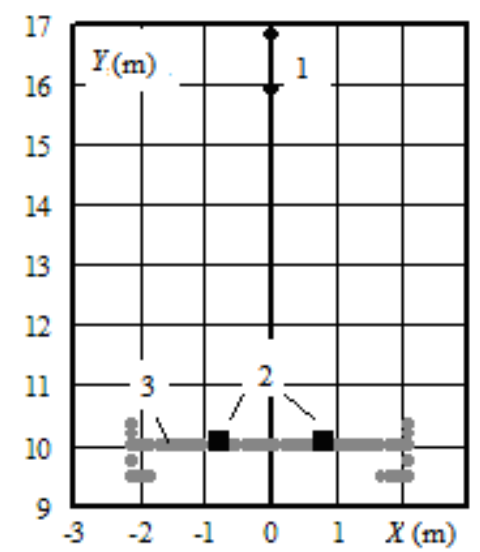

c)

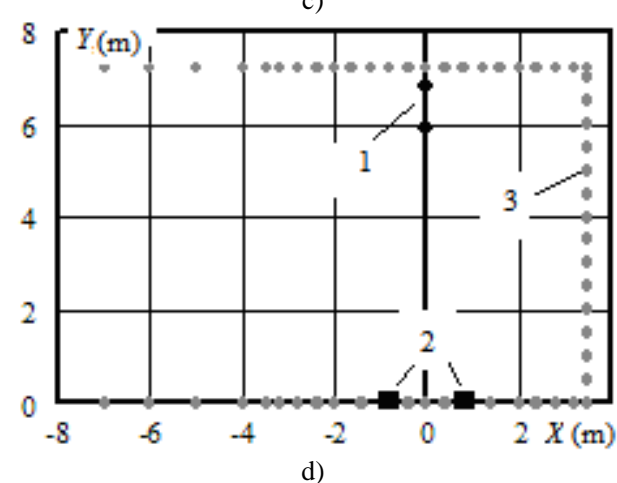

Fig. 4. Coordinates of wires simulating engineering structures: a - tunnel; b - top-road bridge; $\mathrm{c}$ - bottom-road bridge; $\mathrm{d}$ - gallery; 1 - catenary wires; 2 - rails; 3 - wires simulating the profile of engineering structure



a)

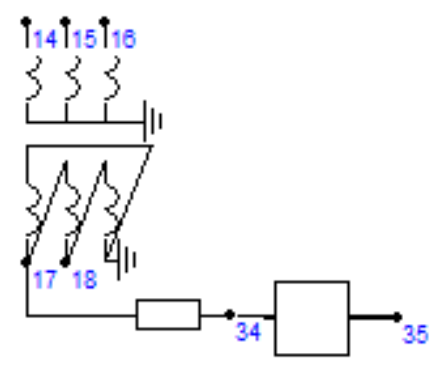

b)

Fig. 5. Layouts of design models:

$\mathrm{a}$ - for flat earth; $\mathrm{b}$ - for the tunnel, gallery and bridges

The current distribution analysis shows the unevenness of the currents in the tunnel and galleries and their comparative uniformity for the metal parts of the bridge. The currents of the conductors of the tunnel and gallery models are greater in those conductors, which are located closer to the contact network wires. If these currents are in good condition, they reach tens of amperes.

The results of electric and magnetic fields calculations, as well as the Poynting vector at the height of the observation points $1.8 \mathrm{~m}$ from the railroad track are shown in Table 1 and Fig. 6 - 12. The maximum values of the components $E_{\mathrm{X}}$ and $H_{\mathrm{Y}}$ are given for the range of coordinate $X$ changes within the range from $-2 \mathrm{~m}$ to $+2 \mathrm{~m}$.

\section{TABLE I MAXIMUM VALUES OF EMF COMPONENTS}

\begin{tabular}{|c|c|c|c|c|c|c|}
\hline \multirow{2}{*}{ Railway condition } & \multicolumn{3}{|c|}{$\boldsymbol{E}, \mathbf{k V} / \mathbf{m}$} & \multicolumn{3}{c|}{$\boldsymbol{H} / \mathbf{m}$} \\
\cline { 2 - 7 } & $\boldsymbol{E}_{\boldsymbol{X}}$ & $\boldsymbol{E}_{\boldsymbol{Y}}$ & $\boldsymbol{E}_{\text {MAX }}$ & $\boldsymbol{H}_{\boldsymbol{X}}$ & $\boldsymbol{H}_{\boldsymbol{Y}}$ & $\boldsymbol{H}_{\text {MAX }}$ \\
\hline Flat & 0.29 & 1.86 & 2.63 & 34.24 & 4.08 & 48.44 \\
\hline Gallery & 0.41 & 0.82 & 1.17 & 8.71 & 4.48 & 12.39 \\
\hline Top-road bridge & 0.05 & 2.06 & 2.92 & 34.05 & 2.62 & 48.16 \\
\hline Bottom-road bridge & 0.45 & 0.84 & 1.18 & 9.88 & 4.81 & 13.97 \\
\hline Tunnel & 0.61 & 1.07 & 1.51 & 5.65 & 3.26 & 7.99 \\
\hline
\end{tabular}
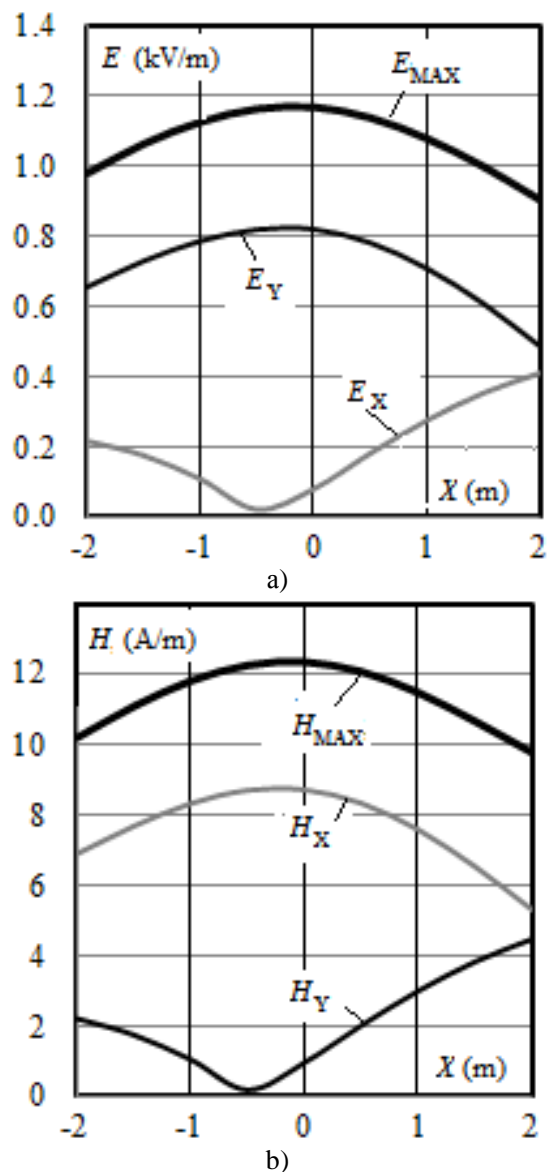

Fig. 6. Dependence of EMF strengths on $X$-coordinate for the gallery: $a$ - electric field; $b$ - magnetic field 

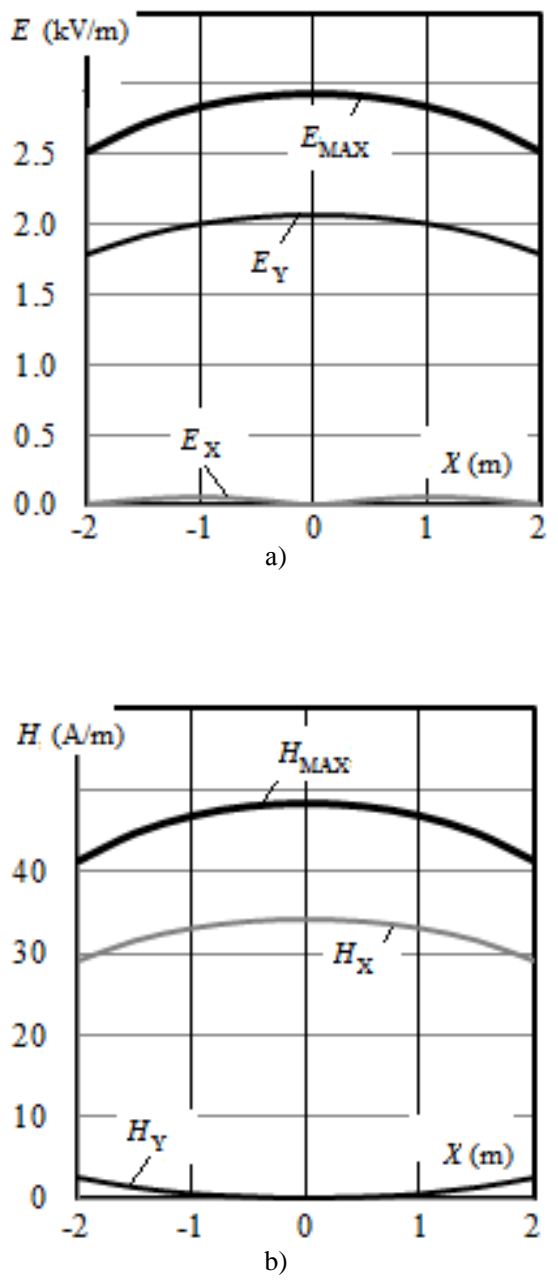

Fig. 7. Dependence of EMF components on $X$-coordinate on the top-road bridge: $a$ - electric field; $b$ - magnetic field

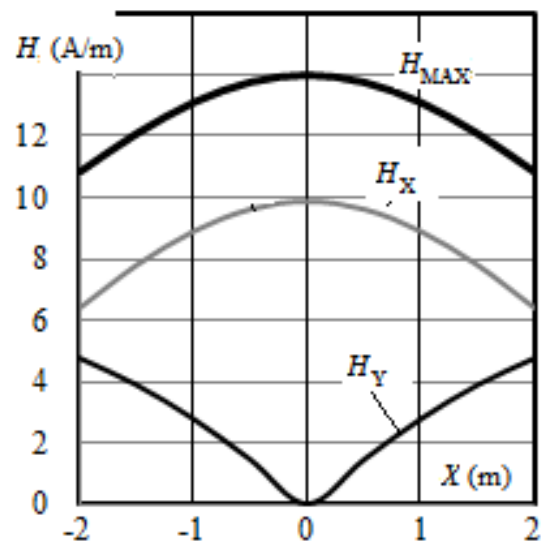

b)

Fig. 8. Dependence of EMF components on $X$-coordinate for the bottom-road bridge: a - electric field; $\mathrm{b}$ - magnetic field

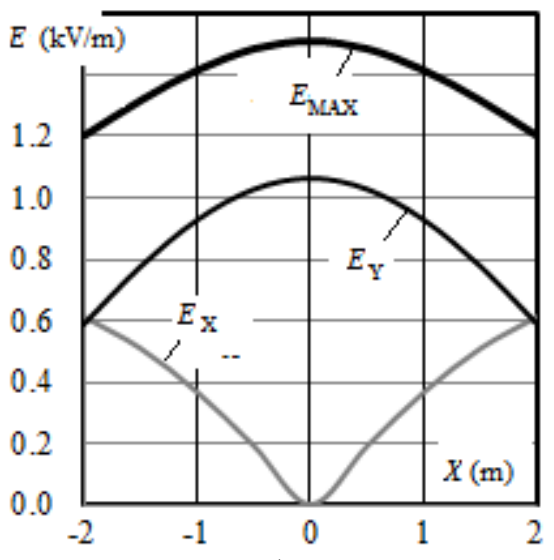

a)

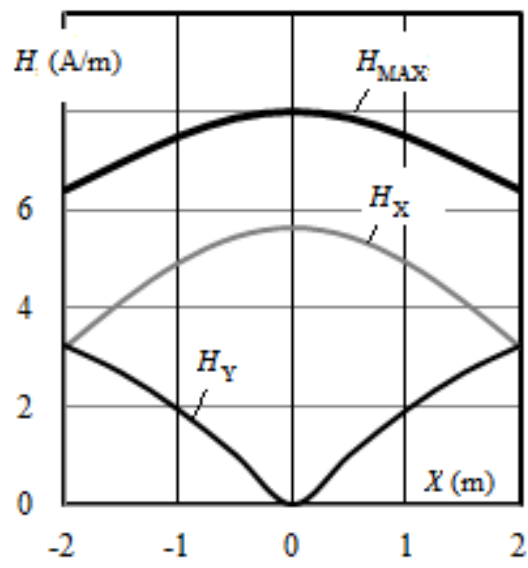

Fig. 9. Dependence of EMF components on $X$-coordinate in the tunnel: a electric field; $b$ - magnetic field 


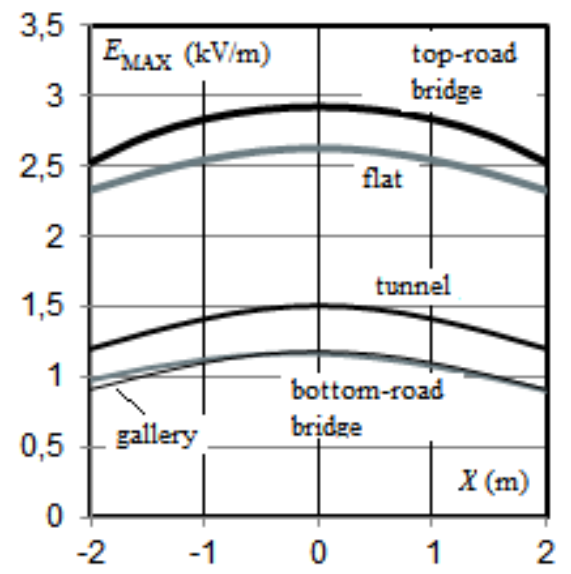

a)

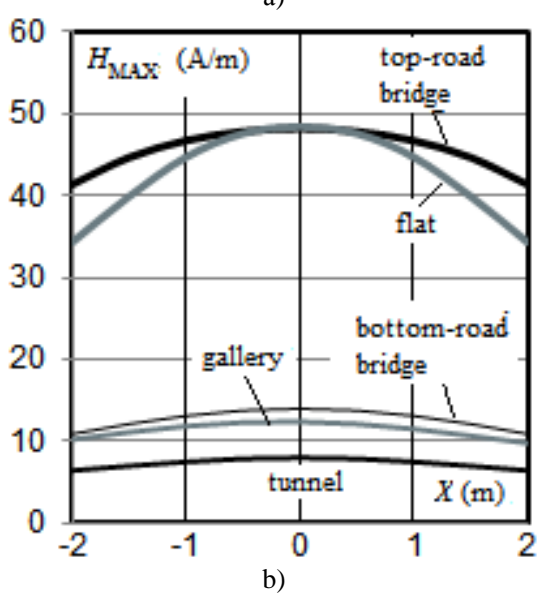

Fig. 10. Summary diagram of EMF dependence on $X$-coordinate: $\mathrm{a}$ - electric field; $\mathrm{b}$ - magnetic field
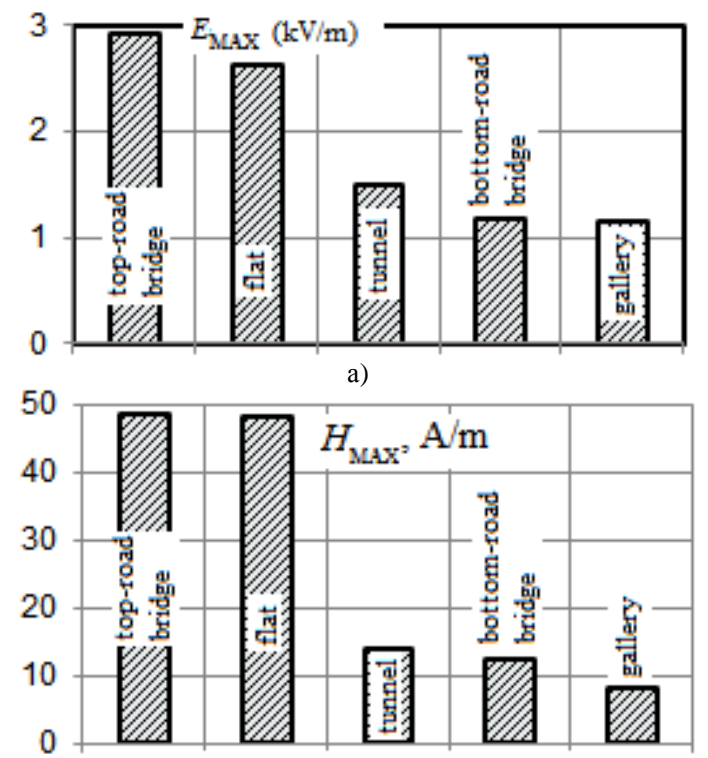

b)

Fig. 11. Comparison of EMF maximum amplitude values: $a$ - electric field; $b$ magnetic field

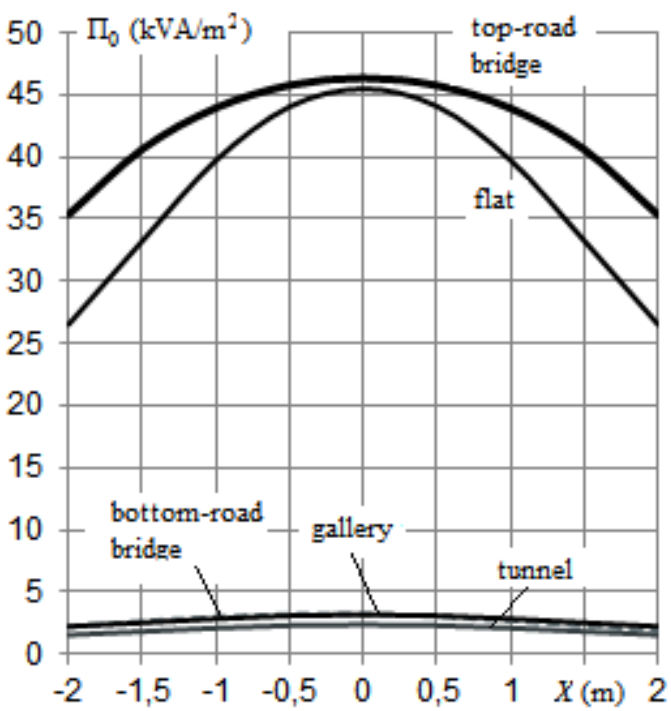

Fig. 12. Comparison of flux density of electro-magnetic energy

The results of electromagnetic fields modeling in railway engineering structures show that the top-road bridge has field strengths higher than those in the open space at the height of $1.8 \mathrm{~m}$ from the railway track. Tunnel, a bottom-road bridge and a gallery are characterized by less strength and lower fluxes of electromagnetic energy. This is due to the proximity to the contact network of shielding metal elements of these engineering structures. The energy transfer by the electromagnetic field in tunnels, galleries and bottom-road bridges occurs in a confined space between the contact network and nearby grounded structures.

\section{CONCLUSION}

The presented method for modeling electromagnetic fields in railway engineering structures - galleries, bridges, tunnels allows obtaining values of the field strengths corresponding to the current mode of the traction power supply system. The presence of grounded conducting objects of engineering structures significantly changes the spatial distribution of the field in comparison with open space with flat relief of ground surface. The strongest electric and magnetic fields are created by electrified railway on top-road bridges.

\section{References}

[1] S.M. Apollonsky, Problems of electro-magnetic safety on electrified railway. 2 vols. Volume II. Electromagnetic safety on railway with alternating current in the traction network. Moscow: RUSAINS, 2017.

[2] S.M. Apollonsky, A.N. Gorsky, Calculations of electromagnetic fields. Moscow: Marshrut, 2006.

[3] V.P. Zakaryukin, A.V. Kryukov, N.V. Buyakova, «Improvement of Electromagnetic Environment in Traction Power Supply Systems», The power grid of the future, Otto-von-Guericke University Magdeburg, pp. 39-44, October 2013

[4] A. Steimel, Electric traction motive power and energy supply. Munchen: Oldenbourg Industrieverlag, 2008.

[5] H. Biesenack, G. George, G. Hofmann, A. Schmieder, E. Braun, K. Girbert, R.C. Klinge, R.Puschmann, S. Röhlig, E. Schlechter, E. Schneider, A. Stephan, G. Zimmert. Energieversorgung elektrischer Bahnen. Teubner Verlag. Wiesbaden, 2006. 
[6] A. Ogunsola, A. Mariscotti, Electromagnetic Compatibility in Railways, Springer, 2013

[7] A. Ogunsola, U. Reggiani, L. Sandrolini, «Modelling Electromagnetic Fields Propagated from an AC Electrified Railway Using TLM», pp. 567570, June 2009 [International Symposium on Electromagnetic Compatibility, 2009].

8] R.J Hill, «Modelling and simulation of electric railway traction, track signalling and power systems», Transactions on the Built Environment, Vol. 6, pp. 383-390, 1994
[9] M. Mandić, I. Uglešić, V. Milardić, «Design and testing of $25 \mathrm{kV}$ ac electric railway power supply systems», Tehnički vjesnik 20, pp. 505 509, March 2013.

[10] V.P. Zakaryukin, A.V. Kryukov, «Multifunctional Mathematical Models of Railway Electric Systems», pp. 504-508, October 2008 [Innovation and Sustainability of Modern Railway, p. 645, 2008]. 